\title{
Enabling Simultaneous Cooling and Data Transmission in the Terahertz Band for Board-to-Board Communications
}

\author{
Vitaly Petrov ${ }^{\mathrm{a}, *}$, Joonas Kokkoniemi ${ }^{\mathrm{b}}$, Dmitri Moltchanov ${ }^{\mathrm{a}}$, Janne Lehtomäki ${ }^{\mathrm{b}}$, Yevgeni Koucheryavy ${ }^{\mathrm{a}}$ \\ ${ }^{a}$ Department of Electronics and Communications Engineering, \\ Tampere University of Technology, Tampere, Finland \\ ${ }^{b}$ Centre for Wireless Communications, University of Oulu, Finland
}

\begin{abstract}
A system enabling simultaneous cooling and board-to-board communications is proposed and analyzed. It is shown that hollow pipes used in computer cooling systems can be applied for communications with extreme data rates at distances up to tens of centimeters. This is done by using wireless communications in the terahertz frequency band, $0.1-10 \mathrm{THz}$. The experiments were performed in order to observe how straight and curved pipes of different diameters and lengths affect $\mathrm{THz}$ signals propagating inside the pipes. The measured pulses were recorded and used in numerical evaluation of bit error rate and throughput taking into account the effect of all possible combinations of $N$ previous symbols. The numerical results show the dependency of the intersymbol interference on the delay profile of the channel and on the symbol period. The results demonstrate that even with simple on-off keying modulation the throughput reaches few terabits per second with qualitatively low bit error rates. This enables communications between rate-hungry electronics inside computers such as central and graphical processing units while simultaneously providing the cooling functionality.
\end{abstract}

Keywords: board-to-board communication, terahertz band communications, computer architecture, cooling pipes

\section{Introduction}

Following the demand for further miniaturization of consumer electronics, the density of the components inside the computer is constantly growing with every generation [1]. Consequently, the layout of components inside the computers becomes more and more complicated, raising the challenge to provide efficient communications between various intra-computer components, such as central and graphical processing units (CPU, GPU), memory modules (such as RAM) and networking interfaces. These studies are usually performed under the umbrella of board-toboard (B2B) communications and have recently attracted significant interest from the research community [2].

In modern computers, components are usually connected using multilane buses that ensure high and reliable data rates offered by the physical connections between the modules. At the same time, deployment of many buses requires significant physical space inside the computer, thus, complicating scaling of the system. Moreover, the physical connections restrict the component locations and the overall size of the boards. Alternative proposals based on fiber optics (see, e.g. [3, 4, 5]) enable high data rates but are characterized by complex networking infrastructure as they require opto-electronic converters/multiplexors. Another weak point of the fiber optics is the physical space to be reserved for the connectors and the fibers themselves.

\footnotetext{
* Corresponding Author.

Email addresses: vitaly.petrov@tut.fi (Vitaly Petrov), joonas.kokkoniemi@ee.oulu.fi (Joonas Kokkoniemi), dmitri.moltchanov@tut.fi (Dmitri Moltchanov),

jannel@ee.oulu.fi (Janne Lehtomäki), yk@cs.tut.fi (Yevgeni Koucheryavy)
}

Open space wireless solutions, enabled by the appearance of the miniature wireless transceivers working in the mmWaves spectrum, have been recently proposed as an alternative to conventional "wired-in-nature" metallic or fiber connectors $[6,7]$. These systems are featured with the advantages of wireless communications, including multicasting and broadcasting capabilities, and provide the data rates, sufficient for the intra-computer traffic with relatively low intensity [8]. These solutions also bring certain freedom in the components locations, allowing for more efficient use of the limited space and the more compact 3D layouts [9]. However, the majority of extensive intracomputer communications (e.g. chipset to GPU, CPU to RAM, etc.) are inherently point-to-point, and does not benefit from the multicasting or broadcasting features, brought by the open space wireless design. On the contrary, harmful effects of interference and, thus, the need for efficient medium access control at extremely high rates to arbitrate the concurrent transmissions make the open space wireless solutions problematic in practice. The applicability of frequency division schemes is also limited due to the the link capacity decreasing with narrowing the channel bandwidth. As a result, a technique to keep the independent links constantly separated is needed.

Another challenging design issue with modern electronics is efficient cooling. The amount of heat to be dissipated constantly grows, thus, calling for more efficient cooling mechanisms than regular fans. In particular, cooling pipes, that originally appeared in large servers, are now incorporated in tablet PCs [10] and even smartphones [11]. Although cooling pipes have good performance, they require substantial amount of space leaving less room for computing, storage and communication components. 
In this paper, we address the above mentioned issues by proposing a system with simultaneous cooling of computer components and wireless data transmission between them. The hollow pipes used in cooling systems are utilized as a transmission medium for wireless communications in the terahertz ( $\mathrm{THz}$, namely, 0.1-10 THz) band. To evaluate the transmission performance of the system we carry out the measurement campaign. The results are then processed using the specifically developed methodology to obtain the bit error rate (BER) and link throughput. Our experiments show that few terabits-per-second (Tbps) throughput over distances up to tens of centimeters could be achieved with a simple on-off keying (OOK) modulation for both straight and curved pipe configurations with fairly low BERs. The achieved performance results proves the ability of the proposed system to replace almost any kind of existing board-to-board data buses increasing the amount of open space in future devices.

The reason to use a specific performance evaluation methodology is that conventional BER estimation techniques are based on the derivation of the signal-to-noise ratio (SNR) [12], which requires the knowledge of the pathloss and assumes the additive white Gaussian noise (AWGN) at the receiver. Calculating the path loss inside a pipe is a complex task as the reception is affected by the intersymbol interference (ISI). Furthermore, the channel is not time-invariant, as the current channel state is affected by the previously transmitted symbols, preventing from using the conventional BER estimation approaches [13]. Therefore, instead of analyzing the reception process analytically, based on the average channel state, we calculate the values of BER for all the important channel states using a combinatorial algorithm, and then estimate the average BER as a weighed sum of these values.

The idea to apply the $\mathrm{THz}$ band for wireless board-toboard and chip-to-chip communications is not novel and has been already discussed in a number of research articles $[14,15,16,17]$ and industrial contributions [18, 19, 20]. Meanwhile, the concept of joining the $\mathrm{THz}$ wireless $\mathrm{B} 2 \mathrm{~B}$ communications with the cooling systems and, thus, avoid the inherent problems of open space solutions rather than mitigate them, have not been comprehensively studied so far. To the best of our knowledge, this is the first work presenting the conceptual design for such kind of system aligned with real measurements and the link-level performance evaluation.

The main contributions of this work are as follows:

- A concept design of a joint cooling and information transmission system for board-to-board communications operating in $\mathrm{THz}$ frequency band: The proposed concept allows to decrease the amount of space inside the computer reserved for the intra-device communications and also, by design, physically separate the independent links (e.g., chipset-to-GPU and CPU-to-RAM) from each other, thus, avoiding the interference problem, which might appear in open space wireless board-to-board communications. The use of extensive $\mathrm{THz}$ band, on its turn, ensures that the link has enough capacity for any possible type of intra-device communications. The selection of the most suitable materials for the pipes and the most effective coolant is also performed.

- Performance evaluation methodology for in-pipe wireless communications: A hybrid methodology, consisting of both the field measurements of $\mathrm{THz}$ propagation through the hollow pipes and a combinatorial algorithm to estimate the bit-error-rate (BER), is designed. The developed methodology allows to analyze the transmission performance of cooling pipes and characterize the trade-offs between BER and throughput for OOK modulation scheme.

- Numerical performance evaluation of THz band inpipe wireless communications: The developed performance evaluation methodology is applied to estimate the major characteristics of the $\mathrm{THz}$ communications over the cooling pipes. In particular, it is shown that for typical communications distances of few centimeters and realistic BER threshold (less than $10^{-6}$ ) the rate achieved by the proposed system depends on the curvature of the pipe and vary between 0.5 and 3 Tbps.

The remainder of the paper is organized as follows. The system design for simultaneous wireless transmission and cooling is introduced in Section 2. Background on $\mathrm{THz}$ propagation is given in Section 3. Cooling techniques and their effects on $\mathrm{THz}$ band wireless communication are discussed in Section 4. In Section 5, casing material selection for the hollow pipes is studied. Wireless communication performance for $\mathrm{THz}$ signals in hollow pipes is evaluated in Section 6, based on actual experiments conducted on $\mathrm{THz}$ propagation in hollow pipes. Conclusions are drawn in the last section.

\section{The Proposed System Design}

The conventional design for communication among various devices residing on or connected to the motherboard is conceptually illustrated in Fig. 1(a). From the communication point of view, all the interfaces are multi-lane buses. For example, Quick Path Interconnect (QPI) and HyperTransport interfaces used by Intel and AMD, respectively, for connecting hardware components (e.g. CPUs) with each other could provide the rates of up to few hundreds of Gbps (400 Gbps over 20+20 lanes operating at $3.2 \mathrm{GHz}$ for QPI). PCIe v4.0 interface standardized in 2015 offers 15.755 Gbps per lane amounting up to $252.064 \mathrm{Gbps}$ over 16-lanes using aggressive 128b/130b encoding. In addition to communications interfaces, Fig. 1(a) also shows the cooling system used in modern computers. It features one or a few fans and heat sinks, and metallic heat pipes going through a number of components that require cooling. The 


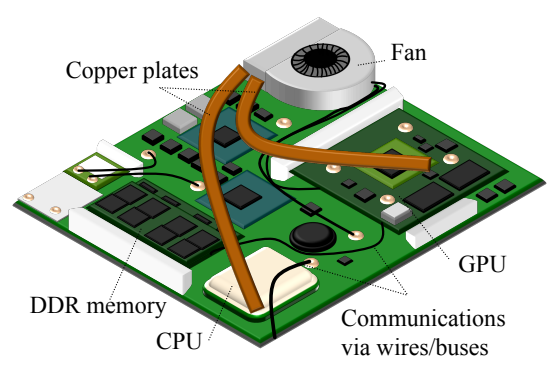

(a) Conventional design

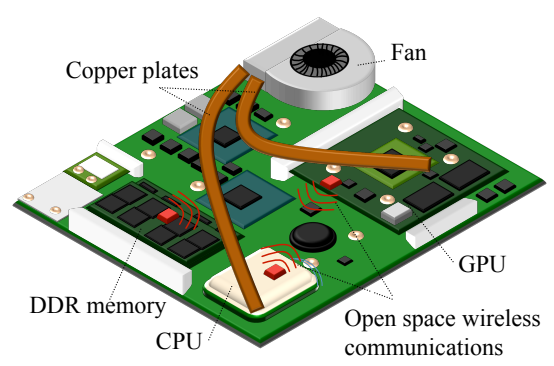

(b) Open space wireless design

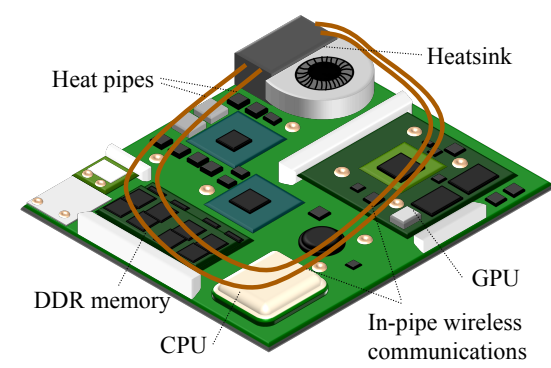

(c) Proposed design

Figure 1: Conventional, open space wireless and the proposed cooling and transmission systems.

heat pipes transfer heat energy from these components to heat sinks and fans.

The open wireless architecture shown in Fig. 1(b) is based on wireless point-to-point links instead of the wires or multi-lane buses. So, each of the boards is about to be equipped with the high-rate wireless transceivers. This architecture decreases the number and complexity of the physical connections between the boards, thus, simplifying the overall system architecture. At the same time, it brings a number of shortcoming and research challenges to be solved, mostly related to the electromagnetic waves propagation inside the computer and insufficient amount of bandwidth at the air interface. To compete with wired technologies the bandwidth must be extremely high which is hardly possible at frequencies lower than few tens of GHz. This effectively outweighs inherent advantages of wireless open space communications such as broadcasting capability allowing for simple point-to-multipoint connectivity. On the other hand, the use of higher frequencies will induce directional antennas and require line-of-sight between transmitter and receiver, which is hardly available in modern systems with tight integration. Last, but not the least, such open-air wireless links could produce a significant amount of interference to other possible wireless links and, in some cases, even to the computing chips and other electronic components.

To address the identified problems, we propose to use the architecture presented in Fig. 1(c), where cooling functionality is supplemented with information exchange via the same medium (hollow pipes). Although the proposed solution is classified as wireless due to the use of $0.1-$ $10 \mathrm{THz}$ band, it is conceptually similar to the optical one requiring "waveguides" to enable communications between nodes. The use of waveguides ensures the absence of interference between the links. On top of this, the effect of $\mathrm{THz}$ radiation on silicon electronics is not fully understood. Covering of propagation paths ensures that the $\mathrm{THz}$ radiation does not affect the functionality of computer components.

In the proposed design, the points, where the hollow pipe comes to contact with a component or a cooling block attached to it (inlet) and leaves the component (outlet) feature $\mathrm{THz}$ transceivers to enable communications between components of interest. Signals are assumed to not propagate through the cooling blocks. The problem of creating $\mathrm{THz}$ emitters has been extensively addressed in the literature with a number of prototypes available. The most common principle is to use laser to irradiate a certain semiconductors. The size of these antennas could be made small allowing integration with future integrated circuits. Moreover, a number of integrated transceiver designs for lower $\mathrm{THz}$ band (up to $1 \mathrm{THz}$ ) have already been proposed, see e.g. [21, 22, 23, 24]. Performance of the proposed design depends mostly on: (i) the type of cooling technique, (ii) the pipe casing material, (iii) communication distance and pipe curvature.

Due to the use of simple On-Off Keying modulation scheme, the algorithmic complexity of the proposed system is very low, as the signal can be transmitted with a pulse-based transceiver and received with a simple energy detector. Due to the energy consumption and implementation issues, an analog energy detector, consisting of a square-law device, an integrator, a thresholder, and (optional) a bandpass filter, may be preferred to the digital one for the implementation of the proposed system.

The proposed system and optical design are characterized by similar shortcomings including the need for complex multiplexers to enable networking between more than two devices connected directly. Both are best suited for point-to-point communications links. When the number of devices increases, instead of creating a full mesh between them, one may use star-based architecture with multiplexer in the center [25]. In addition to providing communications between involved entities, our system allows for cooling of components.

\section{Background in Terahertz Propagation}

The path loss in $\mathrm{THz}$ band is generally modeled with

$$
L(f, d)=L_{A}(f, d)+L_{P}(f, d),
$$

where $L_{A}(f, d)$ is caused by molecules absorbing $\mathrm{THz}$ radiation when a wave travels in the medium, $L_{P}(f, d)$ is the path attenuation due to e.g. spreading and antenna aperture.

In lower frequencies, the path loss $L_{P}(f, d)$ is conventionally modeled as $C(f) d^{-\alpha(f)}$, where $C(f)$ and $\alpha(f)$ are environment dependent constants, and $d$ is the distance between transmitter and receiver. When the signal propagates in free space, $\alpha(f) \approx 2$; for hollow metallic pipes 


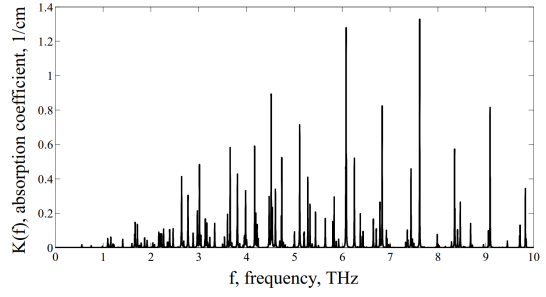

(a) Dry air, $0.76 \mathrm{~g} / \mathrm{m}^{3}$

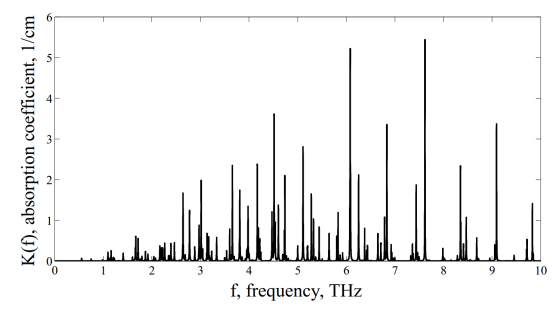

(b) Wet air, $22.86 \mathrm{~g} / \mathrm{m}^{3}$

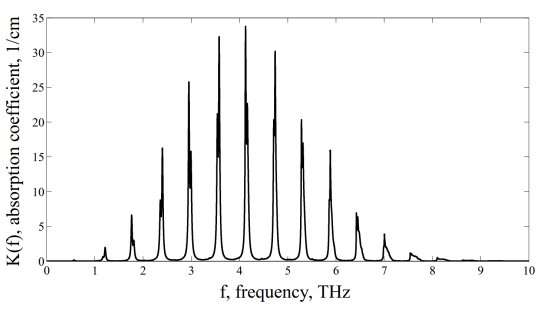

(c) Ammonia

Figure 2: Absorption coefficient for selected media, $0.1-10 \mathrm{THz}, T=273 \mathrm{~K}, p=760 \mathrm{mmHg}$.

$\alpha$ should be between 1 and 2 . The free space propagation loss depends on the chosen band as the path loss (including receiver's antenna aperture) increases following $f^{2}$.

The molecular absorption $L_{A}(f, d)$ is caused by rotational and vibrational movements of molecules. Following the Beer-Lambert law [26] $L_{A}(f, d)=e^{K(f) d}$, where $K(f)$ is absorption coefficient, $d$ is the separation distance [27]. When $K(f)$ is relatively high the absorption-related losses dominate, severely decreasing the received signal strength to the point, where communication is no longer feasible. Fig. 2 demonstrates absorption coefficient, $K(f)$, for dry air, wet air, and ammonia gas $\left(\mathrm{NH}_{3},[28]\right)$ at the standard temperature and pressure conditions, $T=273 \mathrm{~K}$, $p=760 \mathrm{mmHg}$. Wet air has strong absorption in the $\mathrm{THz}$ band. On the other hand, ammonia is characterized by only few absorption lines at isolated frequencies. These lines can be avoided using wise selection of the communication band.

For absorbing media, refractive index is of special importance as it influences the signal propagation in timeverying electromagnetic field. In the $\mathrm{THz}$ band, electromagnetic properties of the signal radiation are frequencydependent and can be expressed using the index of refraction in the following form

$$
\hat{n}(f)=n(f)+i k(f),
$$

where real part, $n(f)$, indicates the phase velocity or refraction of light in the medium and imaginary part $k(f)$ describes attenuation due to absorption loss. Imaginary part, $k(f)$, is also called extinction coefficient and is proportional to the absorption coefficients $K(f)$ via

$$
k(f)=\frac{K(f) c}{4 \pi f}
$$

where $c$ is the speed of light.

\section{Cooling Methods Based on Hollow Pipes}

The proposed system can incorporate different types of cooling including both active and passive. In this section we discuss the applicability of these approaches for the proposed design highlighitng the trade-offs between cooling and transmission performance as well as the overall complexity of the system.

\subsection{Passive Cooling with Hollow Metallic Pipes}

In this method, we rely solely on thermal properties of a hollow metallic pipe for cooling and inside of the pipe is reserved for communication. The pipe can be filled with air or with a gas with no absorption lines in the THz frequency band which can be found by using results in Section III. With this approach wireless communication performance is excellent but cooling performance is weak, so its suitable for cooling the energy.

\subsection{Gaseous Active Cooling}

This method is similar to passive cooling with hollow metallic pipes, however the gas filling the pipe is selected based on its thermal properties and a pump is added for movement of the gas in the pipe. Due to much smaller volumetric heat capacities for gases as compared to liquids, this approach requires large flow speeds but enables favorable propagation conditions for wireless communications. Hydrogen gas has been used as a coolant for turbo generators, it has highest thermal conductance of all gases. This approach is excellent for wireless communication and has somewhat improved cooling performance as compared to fully passive system. Practical experiments related to cooling are out-of-scope in this paper as we focus on wireless communication.

\subsection{Passive Cooling with Heat Pipes}

The heat pipe is passive closed cooling system for transporting heat from the components to a heatsink. Modern laptops can share a heatsink between two components. Heat pipes contain a small amount of working fluid. The fluid evaporates at the thermal source and condenses back to liquid form at the cooler end of the heat pipe. The cooled liquid then travels back to the hot point through a wick causing capillary action. The wireless communication should occur in the vapor cavity. Ammonia shown in the previous section to absorb $\mathrm{THz}$ moderately is one of the popular choices for the working fluid. Actual experiments are needed to account for practical effects such as reflections between the ends of the pipe, effects of the boiling on the wireless signals, and effects of the different wick structures (such as sintered or grooved) on wireless signals. However, in principle, this approach can still provide very good communication performance due to the wick containing the liquid with good heat transfer. 


\subsection{Liquid Active Cooling}

Water cooling is out of the question due to extreme attenuation. Liquids also, in general, strongly absorb $\mathrm{THz}$ frequencies. Non-polar liquids absorb much less than polar liquids [29, Chap. 10][30]. It could be possible to use a non-polar liquid with much less attenuation compared to water, however even with that distances would likely be limited to a few centimeters. This approach is not recommended for wireless communication, unless required communication distances are few centimeters at most.

\section{Pipe Casing Material}

We have two requirements on the choice of casing material for the hollow pipes: (i) it should absorb as little $\mathrm{THz}$ radiation as possible (ii) it should have sufficient thermal conductivity to transfer the heat.

Metals and polymers can serve for the casing of the heat pipe. Polymers are transparent and almost dispersionless at $\mathrm{THz}$ frequencies and, thus, the transmitted signal is dissipated out of the pipe instead of forcing the pipe to serve as a waveguide. To ensure a directional transmission, metals should be used as they are characterized by high reflectivity. The reflectivity of a metal surface is near unity in the $0.1-10 \mathrm{THz}$ band due to high electrical conductivity. Thus, well-polished metal surfaces or metal coating mirrors could act as $\mathrm{THz}$ reflectors [31, 32]. The most commonly used metals for this purpose are gold, silver, copper and aluminum.

Consider the properties of metals in $0.1-10 \mathrm{THz}$ band summarized in Table 1 . These are: electrical conductivity, $\sigma$; penetration depth, $\delta$; and thermal conductivity, $\kappa$. The electrical conductivity influences the reflectivity determining how well the signal is reflected from the inner surface of a pipe. Penetration depth controls the minimum allowable thickness of the material affecting the amount of radiation remaining inside. Finally, thermal conductivity determines the ability of a material to conduct the heat. First two metrics are important for information transfer while the latter one affects the cooling efficiency.

The $\mathrm{THz}$ region is out of optical phonon resonances. Still, the low-energy spectrum tail is a major source of absorption at these frequencies. In general, the dielectric response of vibrational modes decays as frequency decreases in the $\mathrm{THz}$ region [31]. The optical properties in $\mathrm{THz}$ band can be described by the Drude model of conduction,

$$
\sigma(\omega)=\sigma_{0} /(1-i \omega \tau)
$$

where $\sigma(\omega)$ is electrical conductivity, $\omega$ is the angular frequency, $\tau$ is relaxation time, and $\sigma_{0}$ is static conductivity. As the relaxation times of the metals are on the order of $10^{-14} \mathrm{~s}, \omega \tau$ is much less than 1 . Therefore, the Drude model can be simplified as $\sigma(\omega) \approx \sigma_{0}[31,33]$ showing that the electrical conductivity $\sigma$ is independent of frequency.
Table 1: Physical properties of selected metals.

\begin{tabular}{|l|l|l|l|}
\hline Metal & $\sigma, 10^{6} \mathrm{Sm}^{-1}$ & $\delta, \mathrm{nm}$ & $\kappa, \mathrm{W} / \mathrm{mK}$ \\
\hline \hline Gold, $\mathrm{Au}$ & 45.2 & 74.9 & 308 \\
\hline Silver, $\mathrm{Ag}$ & 63.0 & 63.4 & 407 \\
\hline Copper, $\mathrm{Cu}$ & 59.6 & 65.2 & 384 \\
\hline Aluminium, $\mathrm{Al}$ & 37.8 & 81.9 & 209 \\
\hline
\end{tabular}

Reflectivity of metals at normal incidence has the form

$$
R(\omega)=\left|\frac{\hat{n}(\omega)-1}{\hat{n}(\omega)+1}\right|^{2},
$$

where $\hat{n}(\omega)=\sqrt{\mu_{r} \epsilon_{r}}$ is the refractive index, $\epsilon_{r}(\omega)$ is relative permittivity, $\mu_{r}(\omega)$ is the relative permeability of the conductor. Since $\mu_{r}(\omega)$ is approximately one for metals and independent of the frequency, it could be simplified as $\hat{n}(\omega)=\sqrt{\epsilon_{r}}$.

The relative permittivity is given by

$$
\epsilon_{r}(\omega)=\epsilon_{b}+i \frac{\sigma(\omega)}{\epsilon_{0} \omega} \approx i \frac{\sigma_{0}}{\epsilon_{0} \omega},
$$

where $\epsilon_{b}$ is the contribution from bound electrons [31], $\epsilon_{0}$ is the permittivity of the vacuum.

In the $\mathrm{THz}$ band $\sigma_{0} / \epsilon_{0} \omega \gg \epsilon_{b}$ leading to

$$
R(\omega) \approx 1-\sqrt{8 \epsilon_{0} \omega / \sigma_{0}} .
$$

Reflectivity of the considered metals is shown in Fig. 3. One can see that reflectivity only insignificantly deviates from unity with the increase in frequency and nearly equal for all these metals. From the reflectivity point of view, copper and silver are the best metals for our application.

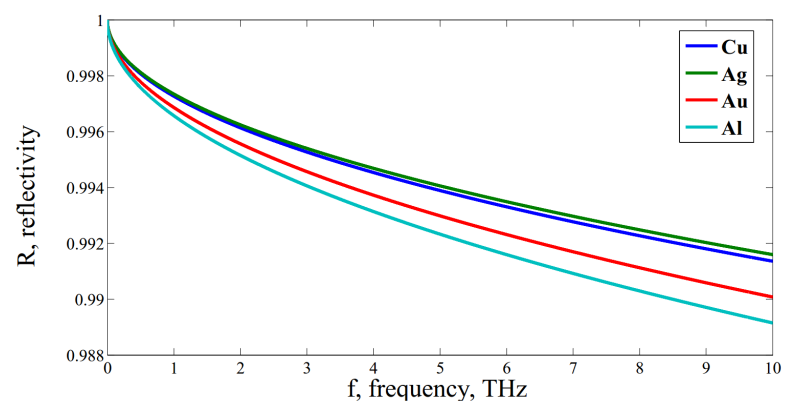

Figure 3: Reflectivity of considered metals.

The thickness of a metal or metal coating should also be taken into account. In order to achieve maximum reflectivity for a given metal, the thickness of the metal layer must be at least two penetration depths, $\delta$ at the frequency of the incident beam [32]. Penetration depth is the attenuation length of exponentially decaying electromagnetic field when the wave is incident on a conductor, that is,

$$
\delta=\sqrt{2 / \omega \mu \sigma},
$$


where $\mu=\mu_{r} \mu_{0}$ is magnetic permeability, and $\mu_{0}$ is the permeability of the vacuum. As demonstrated in Table 1, the penetration depth is less than $100 \mathrm{~nm}$ at $1 \mathrm{THz}$ for common metals. Thus, a few-micron-thick layer is sufficient for an efficient reflector [31].

Finally, consider thermal conductivity, $\kappa$. Thermal conductivity describes the ability of a medium to conduct the heat. Materials of high thermal conductivity are more preferable in heat sink applications as they would allow to take out more heat. Thermal conductivity of common metals is presented in Table 1 . Following these values, the best heat conductors are silver and copper.

Considering all the abovementioned requirements and their values, we see that the silver is the best material for casing of a heat pipe. However, silver and gold are expensive metals. Taking into account the price of materials, copper provides the best trade-off between performance and cost. It also has good characteristics, including high thermal conductivity, and is suitable for the mass-market use. However, we must keep in mind that ammonia (a possible working fluid for heat pipes) is not compatible with copper. If ammonia is used, then aluminum is recommended instead.

\section{Performance Evaluation}

In this section, we perform the proof-of-concept study of the transmission performance of the proposed system. We consider both straight and curved hollow pipes of length $l$, curvature $\alpha$ and diameter $d$. We first describe the testbed used to measure the signals after propagation inside a hollow pipe and then introduce our methodology for BER and throughput analysis of OOK modulation. The results are further reported and discussed. The performance of the associated cooling system can be found elsewhere, the focus here is on wireless communications.

\subsection{Experimental Setup}

In order to evaluate suitable $\mathrm{THz}$ band waveguides, a measurement campaign was conducted. The measurements were made for copper pipes of variable length, diameter and curvature. The measurement device was TeraView Mini Pulse, capable on $\mathrm{THz}$ band measurements from approximately $60 \mathrm{GHz}$ to $4 \mathrm{THz}$, see Fig. 4 . The details of the measurement device are presented in Table 2. The $\mathrm{THz}$ pulse transmissions and reception are based on laser excitation of the antennas. Approximately $100 \mathrm{fs}$ long laser pulses are directed at both, the Tx and the Rx antennas. The laser pulse causes the $\mathrm{THz}$ antennas either to receive, or transmit $\mathrm{THz}$ pulses. In order to capture the time domain behavior of the signal and therefore the frequency domain behavior, the $\mathrm{THz}$ pulses are detected in short time intervals. This time interval corresponds to the resolution time of the signal. To capture the entire pulse, the $\mathrm{THz}$ time-domain spectroscopy (THz-TDS) technique utilizes a delay line for the laser pulses, i.e., the signal reception (or transmission) is delayed one time interval at
Table 2: Characteristics of the the measurement device.

\begin{tabular}{|l|l|}
\hline Device & TeraView MiniPulse \\
\hline \hline Bandwidth & $\sim 4 \mathrm{THz}$ \\
\hline Time resolution & $8.3 \mathrm{fs}$ \\
\hline Frequency resolution & $5.9 \mathrm{GHz}$ \\
\hline Pulse length (laser) & $\sim 100 \mathrm{fs}$ \\
\hline Pulse length $(\mathrm{THz})$ & $<500 \mathrm{fs}$ \\
\hline
\end{tabular}

Table 3: Measurements: "N" - narrow, $10 \mathrm{~mm}$, "W" - wide, $12 \mathrm{~mm}$.

\begin{tabular}{|l|l|l|l|l|}
\hline Copper pipe & $8 \mathrm{~cm}$ & $10 \mathrm{~cm}$ & $12 \mathrm{~cm}$ & $14 \mathrm{~cm}$ \\
\hline \hline Straight & N/W & N/W & N/W & N/W \\
\hline Curved $30^{\circ}$ & - & - & - & W \\
\hline Curved $60^{\circ}$ & - & - & - & W \\
\hline Curved $90^{\circ}$ & - & - & - & W \\
\hline
\end{tabular}

the time over the duration of the desired detection window. This requires about 4000 sent pulses per each detected pulse. In the actual measurements, all the results are averaged over 1000 pulses, i.e., each result is obtained with approximately $4 \times 10^{6} \mathrm{THz}$ pulses. After the time domain signal is obtained, the frequency domain behavior can be obtained by performing the Fourier transform.

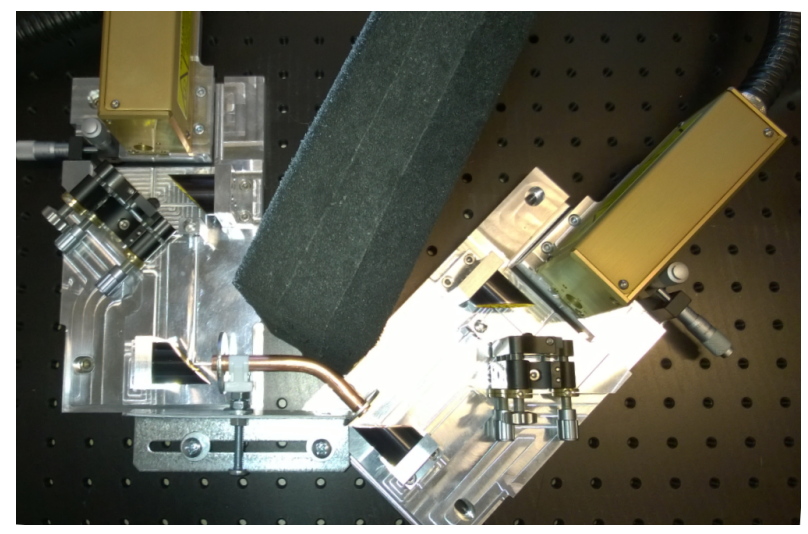

Figure 4: The measurement testbed for curved pipes.

The idea of experiments is to test whether the ordinary pipes can be used as $\mathrm{THz}$ waveguides. To do this, we utilized copper pipes due to their availability, low cost and the widespread use in cooling systems. No special treatment has been applied to the inner surface. Two different diameters were chosen: $10 \mathrm{~mm}$ and $12 \mathrm{~mm}$ diameter pipes, with $8 \mathrm{~mm}$ and $10 \mathrm{~mm}$ inner diameters, respectively. For both thicknesses, a variety of different length pipes were measured. Furthermore, we measured pipes of several curvatures from $0^{\circ}$ to $90^{\circ}$ with $30^{\circ}$ intervals for the $14 \mathrm{~cm}$ long pipes. The measurement cases are summarized in Table 3. The measurements were made in dry air $1.5 \%$ of water vapor), validating the proof-of-concept for the pipe performance. Thus, the presented data provides the lower bound on system performance for passive and gaseous active cooling methods. For more accurate measurements 


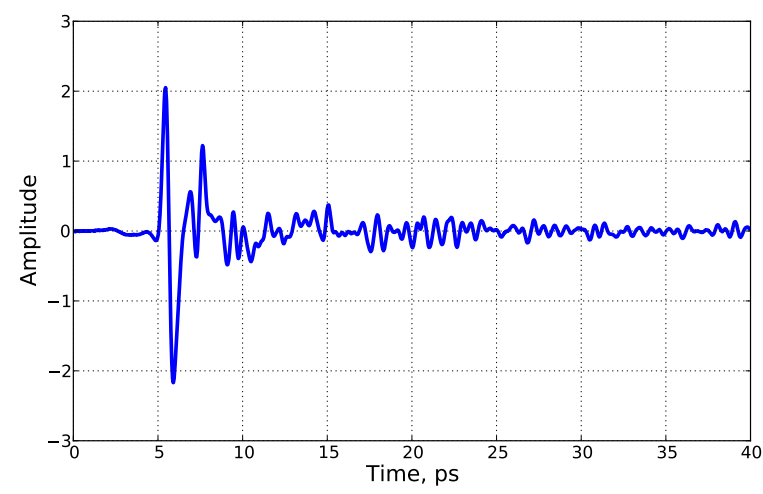

(a) Straight pipe, $12 \mathrm{~cm}$

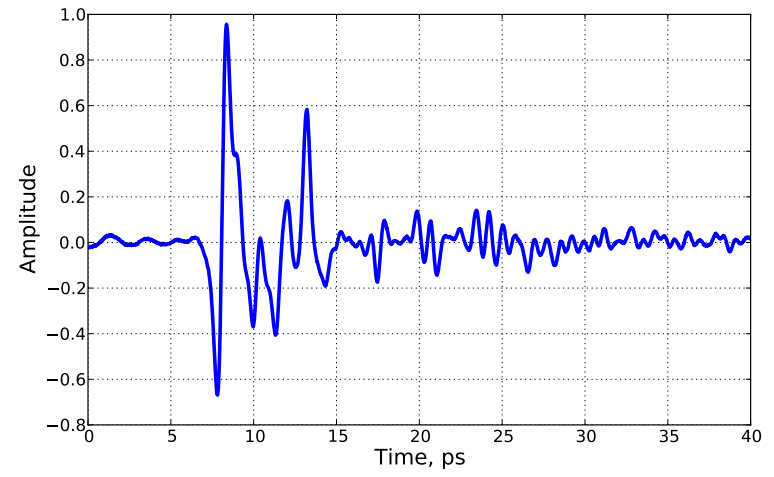

(b) Curved pipe, 90 degrees

Figure 5: Time response of the channel (amplitude is in a.u.).

for heat pipes, wick should be added to the inner surface of the pipe and is left for future work. Also, in real application the liquid will flow in the wick further complicating things. However, the results are still expected to provide rough performance estimation also for the heat pipe. For liquid cooled systems, wireless communication is not feasible unless distances are very short and this cooling method is not modeled here for wireless communication.

Next we will present the obtained results showing that the copper pipes are a viable candidate for $\mathrm{THz}$ waveguides since they (i) efficiently reflect $\mathrm{THz}$ radiation, (ii) are already widely used in cooling applications. It is also shown that the reflections inside the pipes increase the BER. This can be compensated with longer inter-symbol time, decreasing the theoretical data rate at the same time.

\subsection{BER and Throughput Estimation Methodology}

Pulse-based OOK modulation has been suggested as a simplest solution for $\mathrm{THz}$ communications $[34,35]$. In OOK transmitter generates a pulse to send logic " 1 " and keeps silent to send logic " 0 ". At the same time, receiver applies an energy detector to reveal if there was enough energy received to correspond with the transmitted pulse [36]. The results reported below provides the lower bound on the system performance leaving room for further improvements.

In the link level performance assessment we concentrate on two metrics. These are BER and throughput. Observe that assuming no separation distance between pulses and pulse of rectangular shape the theoretical raw throughput for OOK modulation is given by $1 / \Delta t$, where $\Delta t$ is the pulse duration. With our device having $4 \mathrm{THz}$ bandwidth, we cannot reach any value higher than 4 Tbps. Furthermore, if one wants to minimize the error rate at the decoder, one has to provide sufficient separation between symbols, $\tau$, to let the wireless channel "relax" after the previous transmission of logic " 1 ". This effect is highlighted in Fig. 5 illustrating the structure of the channel response to the transmission of the pulse, where the pulse waveform at the receiver is averaged over 1000 realizations of the channel response. This gives the throughput of $1 /(\tau+\Delta t)$.
When the inter-symbol separation, $\tau$, is non-zero, there is a probability that the current symbol will be erroneously decoded. Furthermore, the smaller the $\tau$, the stronger is the inter-symbol interference (ISI). The channel is also non-symmetric implying that the probability of error when receiving either " 0 " or " 1 " after " 0 " is negligible compared to the probability of error when receiving " 0 " after " 1 ". Thus, there is an optimization task of maximizing the system throughput, $S=[(1-B E R) /(\tau+\Delta t)]$, if the channel response at the receiver is known for the sequence " 10 ". To solve this task, we estimate BER for given $\tau$ and $\Delta t$.

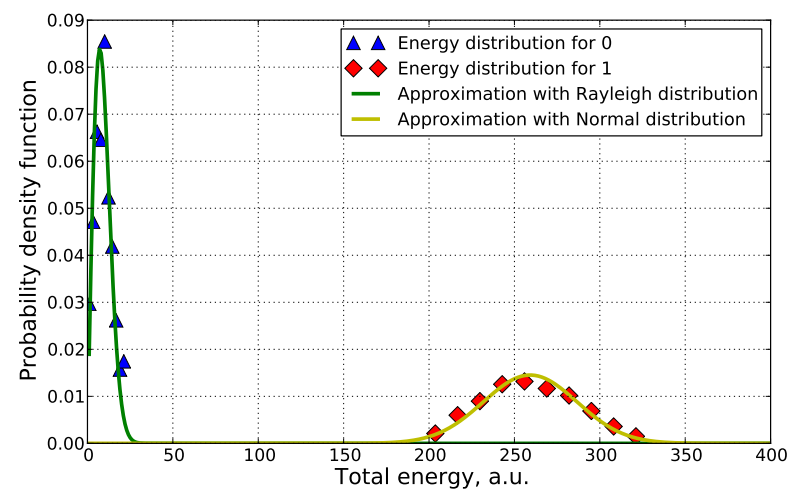

Figure 6: Energy: 1 and 0 symbol energies after 15 previous symbols.

The conventional approach to estimate the BER is based on SNR [12]. However, it requires the knowledge of the path loss and explicitly assumes the additive Gaussian white noise (AWGN) at the receiver. Estimating the path loss inside a pipe of circular configuration is a complex task as there are numerous multi-path components arriving at the receiver shifted in time. Moreover, compared to the conventional AWGN channel, the dominating harmful effect in our case is ISI.

We proceed with computationally intensive approximation. First, observe that time response of the channel we obtained is averaged over a large number of measurements and their amplitude is several orders higher than the noise floor. Secondly, the ISI is much longer than the pulse duration, $\Delta t$, see Fig. 5. Finally, recall that for energybased detector the BER value depends on the threshold 


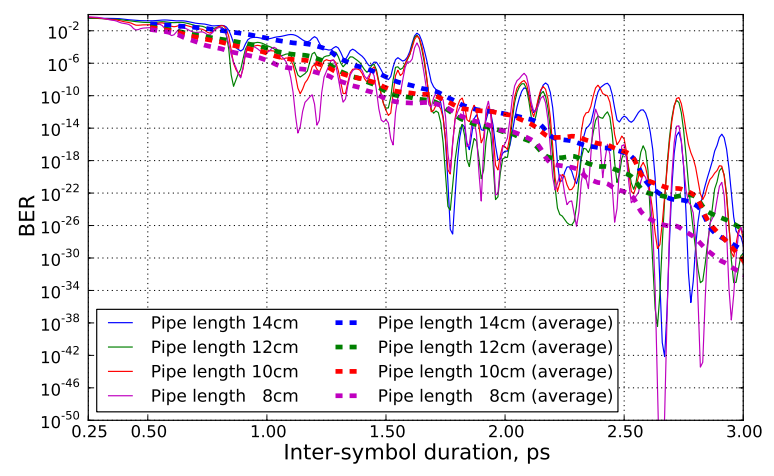

(a) Straight narrow pipe

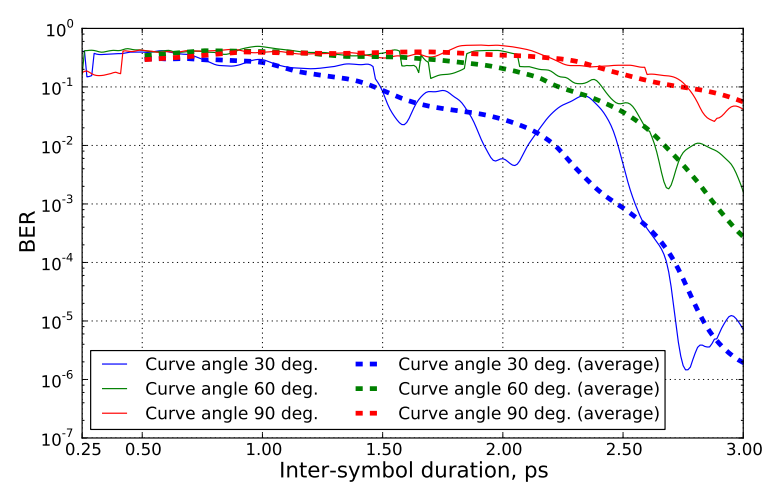

(c) Curved narrow pipe

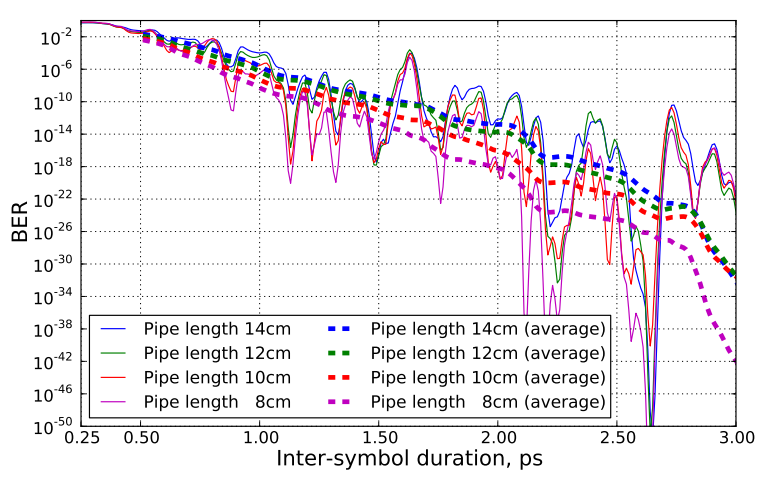

(b) Straight wide pipe

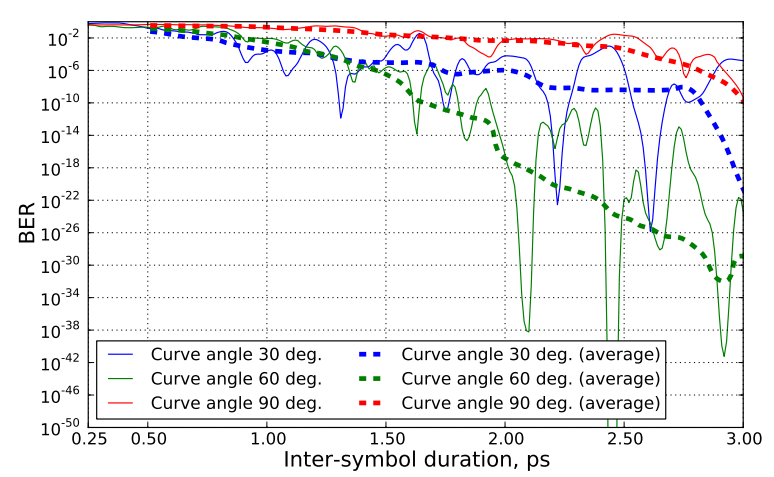

(d) Curved wide pipe

Figure 7: BER for different pipe configurations.

and reaches the optimal value if the threshold is placed at the intersection of probability density functions (pdf) of energy levels, corresponding to symbols "1" and " 0 " [12].

To obtain reliable estimates of pdfs corresponding to "1" and " 0 " the number of measurements shall be on the order of few thousands which is impossible to get in practice. However, assuming equal source and channel coders at the transmitter side, we generate an interfering signal for the symbol of interest for each of the possible previous $2 N$ combinations symbols by superimposing them. Taking this way, for each combination we know the channel response defining ISI. We then add a corresponding useful signal (received pulse for "1" and silence for "0") and estimate the total amount of energy at the receiver. We end up with two arrays of energy levels. Based on these arrays, calculated for all the considered values of $R$, we build empirical pdfs of energy distribution at the receiver for both symbols, see Fig. 6. Despite the fact, the complexity of the proposed algorithm is in the order of $\mathcal{O}\left(2^{N} R\right)$, the complexity of the single run is very low: just get the sum of the array, consisting of $2 N$ elements, which could be done not slower than $\mathcal{O}(N)$. Therefore, the proposed approximation is much more feasible than deriving the pure measurement-based pdfs by obtaining the thousands of measuring samples.

In our study the value of $N$ is set to 15 providing the trade-off between the accuracy of pdf approximation and computational complexity. Since positioning of the emitter and detector are not perfect even for the straight pipes there is no line-of-sight (LoS) and the resulting symbol is superposition of reflected components. Thus, the energy distribution for the symbol " 1 " is approximated by Rayleigh distribution [37]. The ISI for the symbol " 0 " has been found to follow Gaussian distribution for any value of the inter-symbol time. The intersection point between densities providing the optimal value of the energy detection threshold is then found numerically.

\subsection{BER and Throughput}

In this section we present results of BER and throughput estimation for the proposed system. We start with Fig. 7, where the BER is represented as a function of an inter-symbol time, $\tau+\Delta t$. We apply the sliding window average to partially compensate for the fluctuations in the BER caused by the correlation between the current signal and interference from previously received signals. For both wide and narrow pipes we, first, obtain the BER for different lengths of a straight pipe, Fig. 7(a) and Fig. 7(b), and then for different bending angles of a curved pipe, Fig. 7(c) and Fig. $7(\mathrm{~d})$, of the pipe of $14 \mathrm{~cm}$ in length.

Discussing the qualitative effects observed in Fig. 7(a) and Fig. 7(b), we first have to notice that, besides fluctuations, the common trend for BER is to decrease as the inter-symbol intervals increases. In other words, expectedly, when pulses are sent less frequently, the corresponding error rate is lower. Talking about the straight pipe case, specifically, we note that the shorter pipes have lower values of BER for the same inter-symbol intervals. This is 


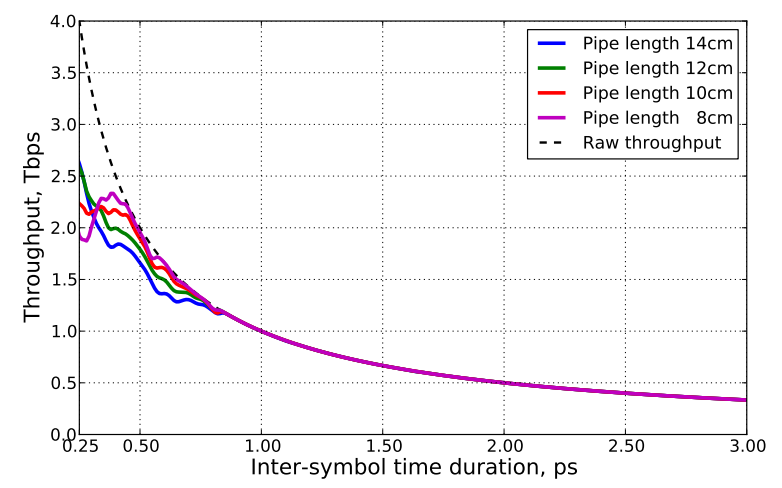

(a) Straight narrow pipe

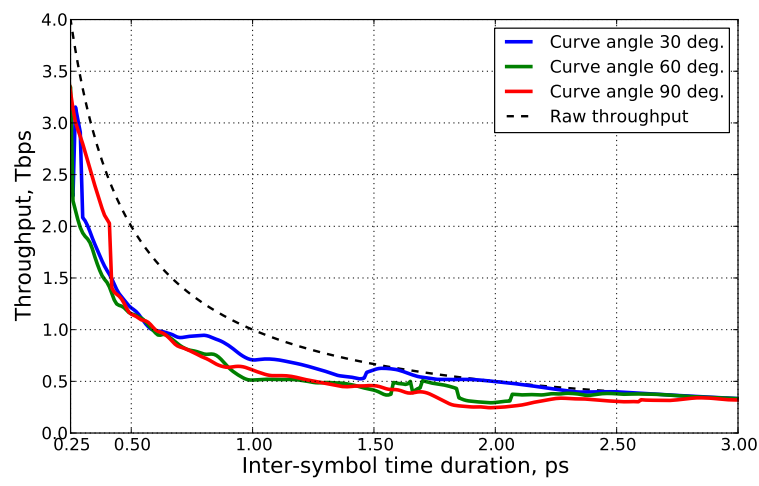

(c) Curved narrow pipe

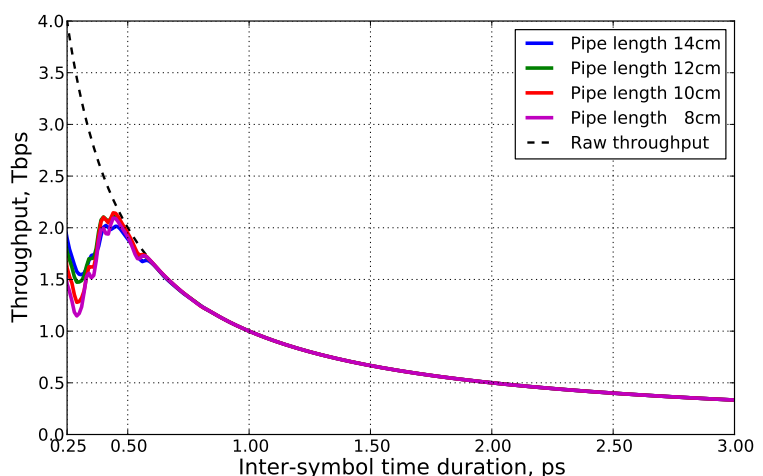

(b) Straight wide pipe

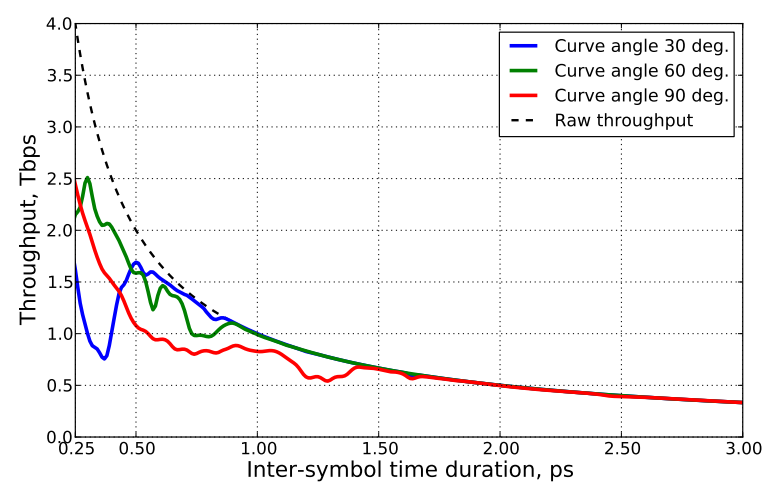

(d) Curved wide pipe

Figure 8: Effective throughput for different pipe configurations.

also logical, as lower lengths lead to fewer reflections and, thus, results in the less attenuated components arriving to the receiver. We recall that $\mathrm{BER}$ values below $10^{-18}$ provides perfect channel conditions ensuring reliable pointto-point communications. Thus, the proposed system has sufficient reliability of the data transfer if straight pipes are used. These value of BER is attained for approximately 2.5-3.0 ns and 2.0-2.5 ns inter-symbol interval ranges for narrow and wide pipes, respectively.

Analyzing the data presented in Fig. 7(c) and Fig. 7(d), we stress that the variability of the obtained results is significantly larger. There are two explanations for this behavior. First, the curvature of the pipe induce complex reflections at the bending point that shall lead to significantly higher variance of the measured channel response and, as a result, require more extensive simulation campaign. Another plausible explanation could be the complexity of experiments themselves, particularly, their repeatability. Indeed, to obtain coherent results perfect bending and further positioning is required. Imperfections in bending of pipes may also lead to special configurations, when rays reflect back to the transmitter. The contributions of these effects are extremely hard to assess without detailed analytical propagation model of $\mathrm{THz}$ waves inside a pipe. Nevertheless, the practical results presented below provide qualitative assessment on the effect of bending, in general, and provide insights on the effect of curvature.

Analyzing Fig. 7(c) and Fig. 7(d) we see that for wide pipe the better BER is obtained for $60^{\circ}$ than for $30^{\circ}$ con- tradicting the expectations. Also, the quantitative difference in BER between curved wide and curved narrow pipes is extremely large, i.e. curved narrow pipe performs much worse, having BER in the range $\left(10^{-7}-0.1\right)$ compared to $\operatorname{BER}\left(10^{-50}-10^{-6}\right)$ for the curved wide pipe case. Besides these two artifacts, we can still benefit from the performed experiments. First, observe that the quantitative values of BER for the curved wide pipe are not much lower than those for the straight wide one. Also, we notice a clear dependence between the bending angle and the associated BER: the less straight is the pipe, the worse would be the BER value. Finally, bending angles of more than $30^{\circ}$ result in high values of BER (more than $10^{-10}$ for reasonable inter-symbol time intervals). Therefore, the proposed system should be used for straight pipes and pipes with low bending angle. If applied for the bended pipes, the system should incorporate channel coding to compensate for high BER.

Table 4: The optimal throughput of the link in Tbps.

\begin{tabular}{|l|l|l|l|l|}
\hline Type/Length & $8 \mathrm{~cm}$ & $10 \mathrm{~cm}$ & $12 \mathrm{~cm}$ & $14 \mathrm{~cm}$ \\
\hline Straight, narrow & 2.51 & 2.52 & 2.53 & 2.56 \\
\hline Straight, wide & 2.17 & 2.15 & 2.12 & 2.03 \\
\hline \hline Type/Curvature & $0^{\circ}$ & $30^{\circ}$ & $60^{\circ}$ & $90^{\circ}$ \\
\hline Curved, narrow, $14 \mathrm{~cm}$ & 3.56 & 3.17 & 2.73 & 3.16 \\
\hline Curved, wide, $14 \mathrm{~cm}$ & 2.03 & 1.7 & 2.49 & 2.38 \\
\hline
\end{tabular}

In addition to the reliability of the proposed system its performance in terms of achievable data rates is also of 
high importance. To investigate this question, we calculate the theoretical throughput value for the given channel as $S=[(1-B E R) /(\tau+\Delta t)]$. The results are demonstrated in Fig. 8. We observe that for high values of inter-symbol interval (starting from 1ps), the effect of incorrect reception of channel symbols on throughput is negligible due to BER values being in the order of $10^{-6}$. So all the calculated curves follow the trend given by the raw throughput. At the same time, when approaching lower values of inter-symbol intervals, the throughput results are affected by two trends: the increase in the raw throughput $1 /(\tau+\Delta t)$ component and decrease in $(1-B E R)$ value due to lower inter-symbol interval. Such combination results in the theoretical throughput having maximum in the interval (0.25-0.5) ps. The corresponding throughput value is around $2 \mathrm{Tbps}$, which exceeds the data rate of current wired interfaces for B2B and is absolutely sufficient for any realistic type of traffic. Finally, the optimal throughput for selected distances and curvature is shown in Table 4.

\section{Conclusions}

We have performed preliminary investigation of the joint cooling and transmission system for board-to-board communications in $\mathrm{THz}$ frequency band, 0.1-10 THz. Our analysis revealed that metals are the best materials for casing of heat pipes as they are characterized by high reflection coefficients allowing for heat pipe to serve as a waveguide. Taking into account the need for good thermal conductivity and competitive price, copper is chosen for the casing.

We performed experimental investigation of the link level performance for straight and curved pipes for OOK modulation using two metrics, BER and throughput. For straight pipes the BER on the order of $10^{-18}$ is achieved for inter-symbol intervals on the order to 2.0-3.0 ps ensuring the peak data throughput on the order of 2 Tbps. The curvature may have drastic effect on the link level performance significantly decreasing the attainable BER for the same length of a pipe and degrading the link throughput. Furthermore, no quantitative conclusions can be made regarding the value of BER due to a number of effects complicating the setup of experiments.

The presented study provided the proof-of-concept for the usage of pipes as a way to provide simultaneous cooling and communications capabilities. There are still a number of engineering problems to be solved before such system may hit the market. Our future investigations will include the development of the theoretical propagation model inside metallic pipes and accurate performance assessment in terms of the data rate for curved pipe configurations.

\section{Acknowledgment}

This work was supported by Infotech Oulu, Academy of Finland SeCoFu project, Academy of Finland FiDiPro program "Nanocommunication Networks" 2012 - 2016, and Nokia Foundation.

\section{References}

[1] R. K. Cavin, P. Lugli, and V. V. Zhirnov, "Science and engineering beyond moore's law," Proceedings of the IEEE, vol. 100, no. Special Centennial Issue, pp. 1720-1749, May 2012.

[2] J. Karedal, A. P. Singh, F. Tufvesson, and A. F. Molisch, "Characterization of a computer board-to-board ultra-wideband channel," IEEE Comm. Let., vol. 11, no. 6, pp. 468-470, 2007.

[3] K. Shikama, S. Asakawa, Y. Abe, S. Yanagi, J. Kobayashi, and T. Takahashi, "Compact multi-fiber receptacle interface for onboard optical interconnection," in 2012 2nd IEEE CPMT Symposium Japan, 2012, pp. 1-4.

[4] A. Yamauchi, Y. Morimoto, T. Enomoto, K. Suzuki, H. Masuda, and T. Ishigure, "Graded-index multimode polymer optical waveguide enabling low loss and high density $3 \mathrm{~d}$ on-board integration," in 2016 IEEE 66th Electronic Components and Technology Conference (ECTC). IEEE, 2016, pp. 490-496.

[5] C. Lin, B. Lu, C. Wang, and Q. Wu, "A $2 \times 40$ gbps wireless communication system using 0.14 thz band oritho-mode transducer," in 2015 40th International Conference on Infrared, Millimeter, and Terahertz waves (IRMMW-THz). IEEE, 2015, pp. $1-2$.

[6] X. Song, L. Landau, J. Israel, and G. Fettweis, "Strong los mimo for short range mmwave communication-towards 1 tbps wireless data bus," in 2015 IEEE International Conference on Ubiquitous Wireless Broadband (ICUWB). IEEE, 2015, pp. $1-5$.

[7] M. Jenning, B. Klein, R. Hahnal, D. Plettemeier, D. Fritsche, G. Tretter, C. Carta, F. Ellinger, T. Nardmann, M. Schroter et al., "Energy-efficient transceivers for ultra-highspeed computer board-to-board communication," in International Conference on Ultra Wideband (ICUWB'15), 2015.

[8] J. Israel, J. Martinovic, A. Fischer, M. Jenning, and L. Landau, "Optimal antenna positioning for wireless board-to-board communication using a butler matrix beamforming network," in Smart Antennas (WSA), 2013 17th International ITG Workshop on, March 2013, pp. 1-7.

[9] G. P. Fettweis, N. ul Hassan, L. Landau, and E. Fischer, "Wireless interconnect for board and chip level," in Design, Automation Test in Europe Conference Exhibition (DATE), 2013, March 2013, pp. 958-963.

[10] V. Pradeep, "How the liquid cooling system works in the Surface Pro 4," Available at http://microsoft-news.com/hereshow-the-liquid-cooling-system-works-in-the-surface-pro-4/, Weblink, Accessed on 27.12.2015, 2015.

[11] R. Wilfred, "Xperia Z5 uses dual heat pipes to prevent overheating," Available at http://mobilesiri.com/sony-xperia-z5-usesdual-heat-pipes-to-prevent-overheating/, Weblink, Accessed on 19.12.2015, 2015.

[12] J. Proakis, Digital Communications. McGraw-Hill, 2001.

[13] F. Hlawatsch and G. Matz, Wireless Communications Over Rapidly Time-Varying Channels. Academic Press, 2011.

[14] H. Song and T. Nagatsuma, "Present and future terahertz communications," IEEE Trans. THz Sci. Technol., vol. 1, no. 1, pp. 256-263, Sept. 2011.

[15] Q. Gu, "Thz interconnect: the last centimeter communication," IEEE Comm. Mag., vol. 53, no. 4, pp. 206-215, Apr. 2015.

[16] S. Rakheja, V. Kumar, and A. Naeemi, "Evaluation of the potential performance of graphene nanoribbons as on-chip interconnects," Proceedings of the IEEE, vol. 101, no. 7, pp. 17401765, July 2013.

[17] S. Abadal, E. Alarcn, A. Cabellos-Aparicio, M. C. Lemme, and M. Nemirovsky, "Graphene-enabled wireless communication for massive multicore architectures," IEEE Commun. Mag., vol. 51, no. 11, pp. 137-143, November 2013.

[18] IEEE 802.15.3d, "Application Requirements Document", IEEE 802.15-14/0304r16, May 2015.

[19] IEEE 802.15.3d, "Technical Requirements Document", IEEE 802.15-14/0309r20, March 2016.

[20] IEEE 802.15.3d, "Channel Modeling Document", IEEE 802.1514/0310-19-003d, March 2016. 
[21] M. Abbasi et al. "Single-chip 220-GHz active heterodyne receiver and transmitter MMICs with on-chip integrated antenna," IEEE Trans. Microwave Theory and Tech., vol. 59, pp. 466-478, Feb. 2011.

[22] C. Jastrow et al., "300 Ghz transmission system," IEEE Electr. Let., vol. 44, pp. 213-214, Feb. 2008.

[23] E. Ojefors, J. Grzyb, Y. Zhao, B. Heinemann, B. Tillack, and U. Pfeiffer, "A $820 \mathrm{ghz}$ SiGe chipset for terahertz active imaging applications," in In Proc. IEEE ISSCC, 2011, pp. 224-226.

[24] Q. Gu, Z. Xu, H. Jian, B. Pan, X. Xu, M. Chang, W. Liu, and H. Fetterman, "Cmos thz generator with frequency selective negative resistance tank," Terahertz Science and Technology, IEEE Transactions on, vol. 2, no. 2, pp. 193-202, 2012.

[25] J.-S. Shen and P.-A. Hsiung, Dynamic Reconfigurable Networkon-Chip Design: Innovations for Computational Processing and Communication. Hershey, PA: Information Science Reference - Imprint of: IGI Publishing, 2010.

[26] D. F. Swinehart, "The beer-lambert law," Journal of Chemical Education, vol. 39, no. 7, p. 333, 1962

[27] M. Jornet and M. Akyildiz, "Channel modeling and capacity analysis for electromagnetic wireless nanonetworks in the terahertz band," IEEE Trans. Wir. Comm., vol. 10, no. 10, pp. 3211-3221, Oct. 2011.

[28] www.cfa.harvard.edu, "Hitran: High-resolution transmission molecular absorption database," Harvard-Smithson Center for Astrophysics, Tech. Rep., 2014
[29] X.-C. Zhang and J. Xu, Introduction to THz Wave Photonics. Springer Science+Business Media, LLC, 2010.

[30] L. V. Chrzanowski, J. Beckmann, B. Marchetti, U. Ewert, and U. Schade, "Capability of terahertz-wave instrumentation for non-destructive testing of liquids," in 10th European Conf. NonDestr. Testing, 2010, pp. 1-8.

[31] Y. Lee, Principles of Terahertz Science and Technology. Springer, 2009

[32] M. Naftaly and R. Dudley, "Terahertz reflectivities of metalcoated mirrors," Appl. Opt., vol. 50, 2011.

[33] K. Bane, G. Stupakov, and J. Tu, "Reflectivity measurements for copper and aluminum in the far IR and the resistive wall impedance in the LCLS undulator," in In Proc. EPAC, 2006, pp. 2955-2957.

[34] J. Jornet and I. Akyldiz, "Femtosecond-long pulse-based modulation for terahertz band communication in nanonetworks," IEEE Trans. Comm., vol. 62, no. 5, pp. 1742-1754, May 2014.

[35] I. Akyildiz, J. Jornet, and C. Han, "TeraNets: Ultra-broadband communication networks in the terahertz band," IEEE Wir. Comm. Mag., vol. 21, no. 4, pp. 130-135, Aug. 2014.

[36] R. Cid-Fuentes, J. Jornet, and E. Akyildiz, I. Alarcon, "A receiver architecture for pulse-based electromagnetic nanonetworks in the terahertz band," in In Proc. ICC, 2012, pp. 49374942.

[37] T. Rappaport, Wireless Communications: Principles and Practice, 2nd ed. Prentice Hall PTR, 2001. 